\title{
Synthesis of Highly Reduced Graphene Oxide for Supercapacitor
}

\author{
Chubei Wang, ${ }^{1}$ Jianwei Zhou, ${ }^{1}$ and Feipeng $D u^{2}$ \\ ${ }^{1}$ Institute of Energy and Fuel, Xinxiang University, Xinxiang, Henan 453003, China \\ ${ }^{2}$ School of Materials Science and Engineering, Wuhan Institute of Technology, Wuhan, Hubei 430073, China \\ Correspondence should be addressed to Chubei Wang; wangchubei@163.com
}

Received 7 June 2016; Revised 2 October 2016; Accepted 24 October 2016

Academic Editor: Giuseppe Compagnini

Copyright (C) 2016 Chubei Wang et al. This is an open access article distributed under the Creative Commons Attribution License, which permits unrestricted use, distribution, and reproduction in any medium, provided the original work is properly cited.

\begin{abstract}
A facile method to synthesize highly reduced graphene oxide in solid phase was developed. The reduced graphene oxide was scarcely prepared in solid phase. Solid substances act as spacers and pillaring agents. Sheets can not be close to each other in reduction process, and sheets agglomeration might not form. After reduction reaction is complete, the spacers and pillaring agents are removed. The average interlayer spacing and surface area of product are bigger than those of reduced graphene oxide. The product has few-layered sheet, and the ratio of carbon to oxygen is high, which might imply that the product is more similar to graphene compared to reduced graphene oxide. The specific capacitance of product is almost three times higher than that of reduced graphene oxide at the same current density.
\end{abstract}

\section{Introduction}

Graphene has created a revolution in the field of nanotechnology $[1,2]$. It shows excellent electrical conductivity and catalysis and cocatalysis and large specific surface area and good thermal conductivity [3-5]. Because of these unique properties, it is expected that graphene can be used as supercapacitor and electrode material. The preparation and characterization of graphene are of great interest to chemists. It can be prepared by many techniques including chemical vapour deposition, epitaxial growth on electrically insulating surfaces, micromechanical exfoliation of graphite using Scotch tape, chemical intercalation-exfoliation, and electrochemical exfoliation techniques $[6,7]$. Among these reported methods, the chemical oxidation-reduction method is the most facile, for its cost-effectiveness and potential for scale-up of production. However, the agglomeration of graphene sheets during reduction is a great concern for facile applications as all the excellent properties are associated with the monolayer graphene structure $[8,9]$. Surface modification of graphene sheets through the insertion of some organic or nonorganic spacers is a solution to prevent agglomeration $[10,11]$. This approach might partially regain the high surface area of monolayer graphene, but these spacers might be impurity for graphene.
Graphene oxide (GO) begins in monolayer form with high area; the extent of surface area losses by restacking during reduction process is very large. In simple reduction process, the surface area of reduced graphene oxide (RGO) decreases quickly as the number of sheets per stack increases [12]. Here, we report the preparation of reduced graphene oxide by pillared method in solid phase. The "pillars" are removed after RGO formed. The mixture of GO and solvent form suspension. GO sheets are reduced; many oxygenated functional groups are lost. With the aid of stirring and Brownian movement, the sheets can freely move, and they can be close to each other. If there are no spacers between monolayer sheets, agglomeration is easily formed, which often lead to layer stacking and even form graphite. GO sheets were dispersed in solid phase, the sheets can not move, and then agglomeration might not form. After reduction reaction is complete, the spacers and pillaring agents are removed, and highly reduced graphene oxide (HRGO) is formed. The reduction process might be like plastic deformation, and sheets might not restack to form graphite.

\section{Experimental Section}

2.1. Chemicals. Pristine graphite was purchased from Qingdao BCSM Co. Ltd. (Qingdao, China), and ammonium 


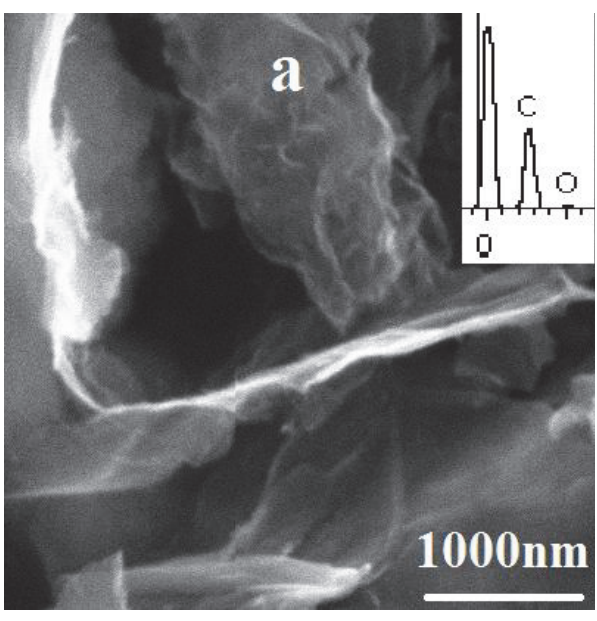

(a)

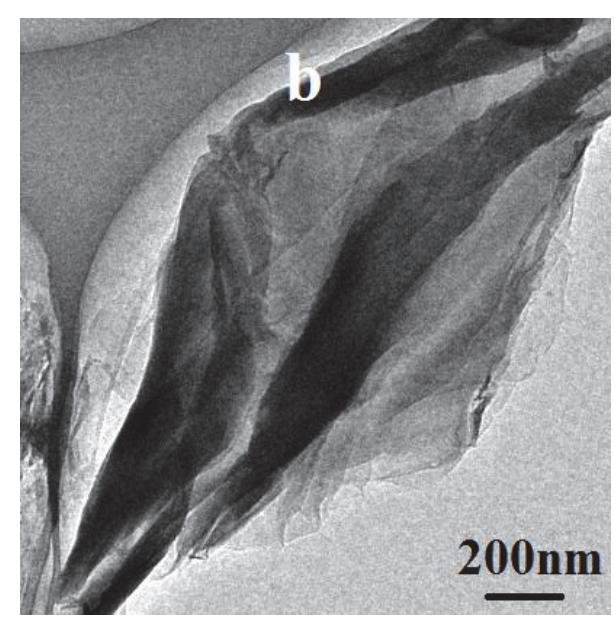

(b)

FIGURE 1: (a) SEM image and EDX spectrum of HRGO. (b) TEM image of HRGO.

chloride $\left(\mathrm{NH}_{4} \mathrm{Cl}\right)$ was supplied by Shanghai Chemical Reagent Company (Shanghai, China). All other chemicals were of analytical grade and were purchased from Beijing Chemical Reagents Company (Beijing, China).

2.2. Characterization. Scanning electron microscopy (SEM) and energy-dispersive X-ray (EDX) spectroscopy were performed using Hitachi S-4800 field emission and FEIQuanta 200 scanning electron microscopes, respectively. High-resolution TEM (HRTEM) was performed on JEOL JEM-2011 electron microscope operated at $200 \mathrm{kV}$, equipped with a Gatan 794 camera. Fourier-transform infrared (FTIR) spectra were obtained on FTS-40 (Bio-Rad, CA, USA). Raman spectra were recorded on Renishaw InVia multichannel confocal microspectrometer with $532 \mathrm{~nm}$ excitation laser. X-ray diffraction (XRD) measurements were obtained on X'pert PRO diffractometer using Co K $\alpha$ radiation. X-ray photoelectron spectroscopy (XPS) with monochromatized Al $\mathrm{K} \alpha \mathrm{X}$-ray $(\mathrm{h} \nu=1486.6 \mathrm{eV})$ radiation (Thermo Fisher Scientific Co., ESCALAB 250, USA) was used to investigate the surface properties of the HRGO. The shift in binding energies was corrected using the $\mathrm{C} 1 \mathrm{~s}$ signal at $284.6 \mathrm{eV}$ as the internal standard. Nitrogen sorption measurements were performed with ASAP 2020 V3.01H (Micromeritics, USA).

2.3. Synthesis of HRGO and RGO. A mixture of GO ( $0.50 \mathrm{~g})$ and water $(50 \mathrm{~mL})$ was sonicated for $1 \mathrm{~h}$. Water bath sonication was performed using JL-60 DTH sonicator (100 W). Ammonium chloride ( $30 \mathrm{~g}, 0.56 \mathrm{~mol}$ ) was added to the GO dispersion at $50^{\circ} \mathrm{C}$ stirring for $2 \mathrm{~h}$, and then it was dried by natural drying method. Then the mixture was calcined at $320^{\circ} \mathrm{C}$ for $1 \mathrm{~h}$, the mixture was cooled down to ambient temperature, and the mixture was washed with water $(20 \mathrm{~mL}$ $\times 3$ ). The HRGO was dried at $50^{\circ} \mathrm{C}$ for $12 \mathrm{~h}$ to yield a black fluffy powder $(0.36 \mathrm{~g})$. Other samples were calcined at 240 , 280 , and $360^{\circ} \mathrm{C}$.

The RGO was prepared according to that of a previous literature [13].
2.4. Capacitance Experiments. The electrode was made by coating $85 \mathrm{wt} \%$ of HRGO and $15 \mathrm{wt} \%$ of acetylene black onto a working electrode. The working electrode was dried at $100^{\circ} \mathrm{C}$ for $10 \mathrm{~h}$. The $\mathrm{CV}$ and $\mathrm{CD}$ of HRGO were measured with $\mathrm{CH} 660 \mathrm{D}$ electrochemical work station using a threeelectrode configuration. Glassy carbon was used as the working electrode, $\mathrm{Pt}$ wire served as the counter electrode, and saturated $\mathrm{AgCl} / \mathrm{Ag}$ served as the reference electrode [14, 15]. All electrochemical measurements were carried out in $0.1 \mathrm{M} \mathrm{Na}_{2} \mathrm{SO}_{4}$ solution as the electrolyte.

\section{Results and Discussion}

3.1. Characterization of HRGO. The morphologies and microstructures are characterized by SEM and HRTEM (Figures $1(\mathrm{a})$ and $1(\mathrm{~b})$ ). The graphene sheets are rather wrinkled, indicating the single-layer and/or few-layer graphene sheets [16]. The monolayer sheets dispersed in solvent and they easily formed wrinkles and twists. These sheets were fixed by solid, and then thermal reduction proceeded. The sheets can not freely stretch. The sheets with wrinkles and twists can not easily form multilayer graphene or graphite. The corresponding EDX spectrum revealed the presence of $\mathrm{C}$ and $\mathrm{O}$ (Figure 1(a)). The peak of oxygen is weak, which implies that $\mathrm{GO}$ is reduced.

The FT-IR of RGO and HRGO are shown in Figure 2(a). HRGO shows the stretching vibrations of $-\mathrm{OH}$ groups at $3420 \mathrm{~cm}^{-1}$, skeletal vibration of unoxidized graphitic domains at $1560 \mathrm{~cm}^{-1}$, and alkoxy C-O at $1180 \mathrm{~cm}^{-1}$. The peak of skeletal vibration of unoxidized graphitic is evident, implying that the HRGO is reduced. RGO shows the stretching vibrations of $-\mathrm{OH}$ groups at $3410 \mathrm{~cm}^{-1}$, skeletal vibration of unoxidized graphitic domains at $1560 \mathrm{~cm}^{-1}$, and alkoxy C-O at $1040 \mathrm{~cm}^{-1}$.

The Raman spectra of HRGO present broadened G band at $1572 \mathrm{~cm}^{-1}$ because of the formation of a conjugated system, in contrast to the G band $\left(1565 \mathrm{~cm}^{-1}\right.$ ) of RGO (Figure 2(b)). The intensity of the D band at $1352 \mathrm{~cm}^{-1}$ of HRGO increases 


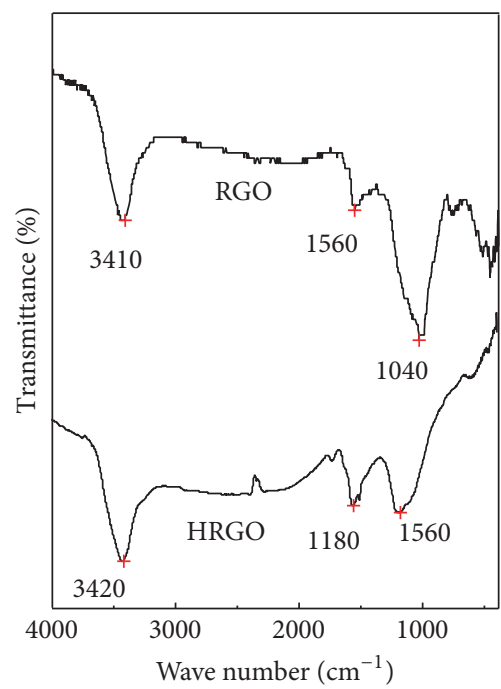

(a)

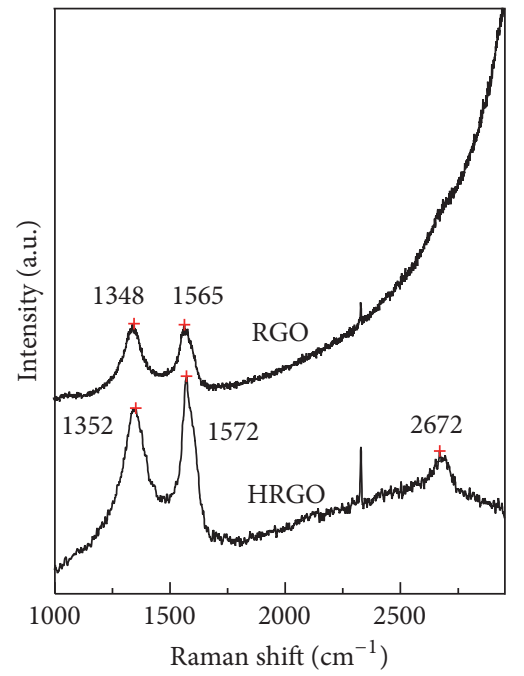

(b)

FIGURE 2: (a) FT-IR spectra of RGO and HRGO. (b) Raman spectra of RGO and HRGO.

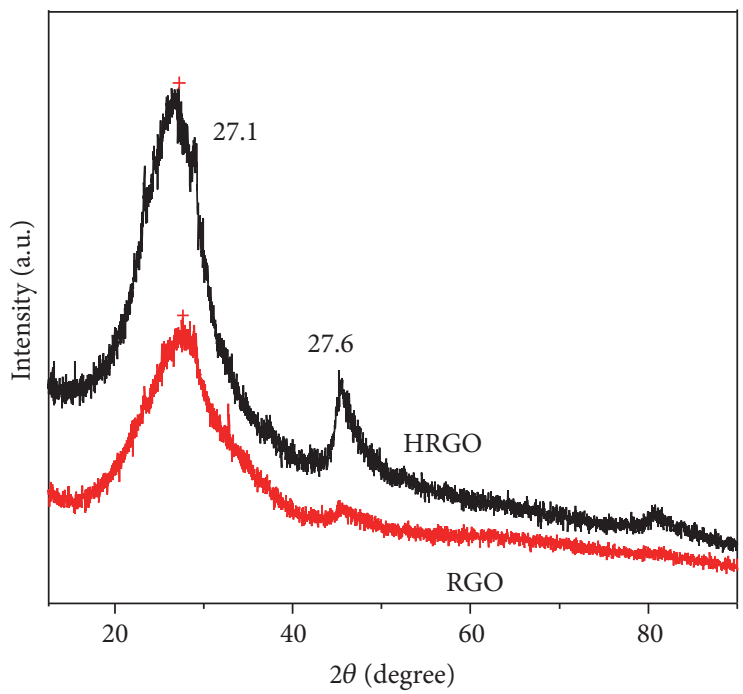

(a)

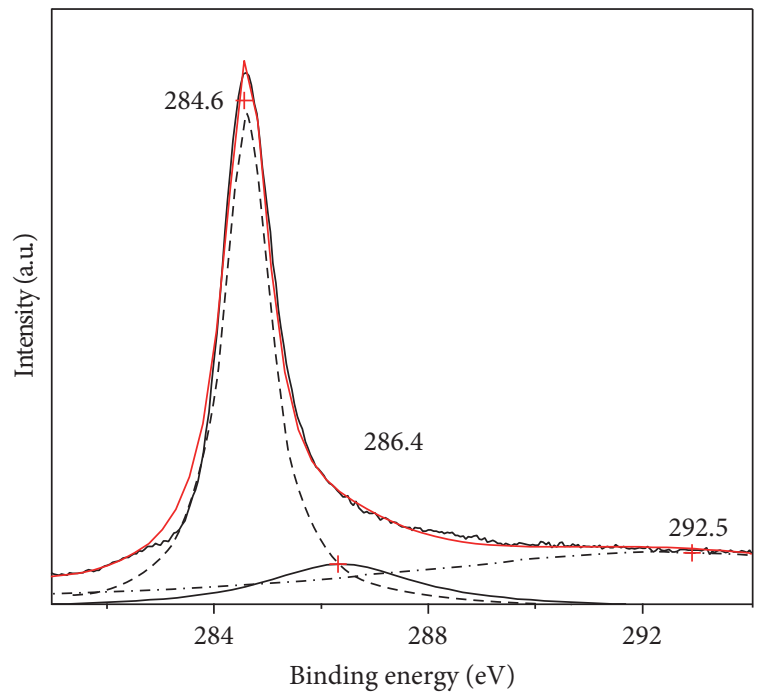

(b)

FIGURE 3: (a) XRD patterns of the RGO and HRGO. (b) Cls XPS of HRGO.

substantially, indicating the decrease in size of the in-plane $\mathrm{sp}^{2}$ domains, possibly due to edges, defects, and disordered carbons [17]. Another intense peak is at $\sim 2672 \mathrm{~cm}^{-1}$, called the $2 \mathrm{D}$ band. The peak is always exhibited by raw graphite, representing the second-order zone boundary phonons. The $2 \mathrm{D}$ band is assigned to the band structure of graphene layers. The $2 \mathrm{D}$ band of graphene is also very useful for the identification of the number of layers as reported by some literatures [18-21]. Upon careful investigation and comparison with the above literatures, the $2 \mathrm{D}$ band of $\mathrm{HRGO}$ suggests the formation of few-layer graphene.

The XRD patterns of HRGO and RGO are shown in Figure 3(a). HRGO exhibits the intensity and broadness of the peak at $2 \theta=27.1^{\circ}$, and the average interlayer spacing of
HRGO is about $0.38 \mathrm{~nm}$. The characteristic peak of RGO is at $27.6^{\circ}(0.36 \mathrm{~nm})$ [22]. The graphene is single-layer or few-layer sheet by micromechanical exfoliation of graphite using Scotch tape; it is in metastable state. If this graphene is squeezed in right direction, it might partly form multilayer graphene or graphite. Some modified graphene types have wider average interlayer space compared to RGO [23-25]. Graphene sheets, with surface modification or functional group, can be squeezed; they can withstand external forces. Though HRGO have few spacers or oxygenated functional groups, the average interlayer spacing of HRGO is wider than that of RGO.

The XPS is also used to analyze the samples of HRGO. The core-level XPS signals of Cls are shown in Figure 3(b). 


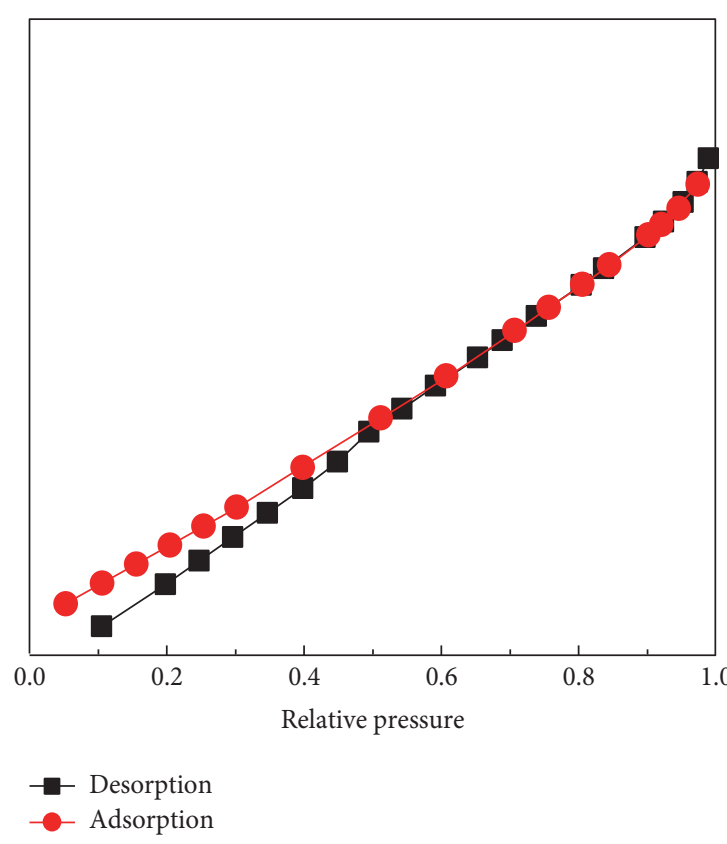

(a)

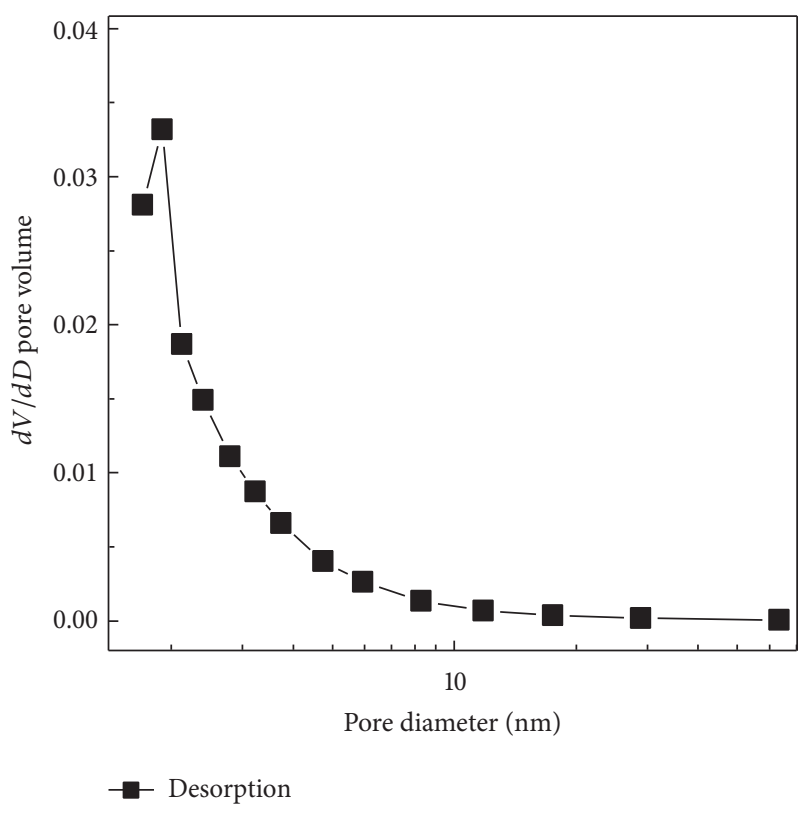

(b)

FIGURE 4: Typical nitrogen adsorption and desorption of HRGO (a) and pore size distribution curves of HRGO (b).

TABLE 1: Atomic concentration of HRGO.

\begin{tabular}{lccc}
\hline Sample & $\mathrm{C}(\%)$ & $\mathrm{O}(\%)$ & $\mathrm{C} / \mathrm{O}$ \\
\hline HRGO & 90 & 10 & 9 \\
\hline
\end{tabular}

The peak centered at approximately $284.6 \mathrm{eV}$ originates from the graphitic $\mathrm{sp}^{2}$ carbon atoms; the peak is strong. The peaks of $\mathrm{C}-\mathrm{O}$ and $\mathrm{C}=\mathrm{O}$ are at 286.4 and $292.5 \mathrm{eV}$, respectively. Compared with the peak of graphitic $\mathrm{sp}^{2}$ carbon atoms, the peaks of $\mathrm{C}-\mathrm{O}$ and $\mathrm{C}=\mathrm{O}$ are weak. In some similar reports about RGO $[13,26]$, the ratio of $\mathrm{C}$ to $\mathrm{O}$ is about 5 . The ratio of $\mathrm{C}$ to $\mathrm{O}$ in HRGO is 9 in Table 1, also indicating that HRGO is highly reduced.

The surface area of HRGO is $468.6 \mathrm{~m}^{2} \mathrm{~g}^{-1}$. The common technique to determine the graphene stack mean layer number is the scaling law:

$$
N=\frac{2600}{A},
$$

where $N$ is number of sheets in a stack, 2600 is the theoretical surface area of graphene in $\mathrm{m}^{2} \mathrm{~g}^{-1}$, and $A$ is the measured BET surface area of the sample in $\mathrm{m}^{2} \mathrm{~g}^{-1}$. According to (1) [12], $N$ is about 6, which might also imply the HRGO is fewlayered sheets. Nitrogen adsorption-desorption isotherm is close to a characteristic IUPAC type IV curve, indicating the presence of pores in the fabricated material, but it is not a typical characteristic IUPAC type IV curve in Figure 4(a). The curve is not closed, and the reason might be that the pores of HRGO are not stable in desorption process. The porous property of HRGO can be further confirmed by pore
TABLE 2: The HRGO degree of reduction with different temperature and yield and their specific capacitances.

\begin{tabular}{lccc}
\hline $\begin{array}{l}\text { Temperature } \\
\left({ }^{\circ} \mathrm{C}\right)\end{array}$ & Yield $(\%)$ & C/O ratio & $\begin{array}{c}\text { Specific } \\
\text { capacitance } \\
\left(1 \mathrm{Ag}^{-1}\right)\end{array}$ \\
\hline 240 & 85 & 7.6 & 160 \\
280 & 81 & 8.2 & 130 \\
320 & 67 & 9 & 128 \\
360 & 53 & 9.4 & 114 \\
\hline
\end{tabular}

size distribution analysis determined by the Barret-JoynerHalenda method in Figure 4(b), from which we can see that there is a distributed peak of micropores centered at $1.9 \mathrm{~nm}$.

3.2. Electrochemical Properties of HRGO. The HRGO degree of reduction with different temperature and HRGO specific capacitances are shown in Table 2. The ratio of carbon to oxygen range between 7.6 and 9.4 was used in the present study. The values of specific capacitance decreased with the increasing ratio of carbon to oxygen from 7.6 to 9. The high specific capacitances were observed at ratio of carbon to oxygen of 7.6-8.2. As for highly reduced graphene oxide (HRGO), the ratio of carbon to oxygen should be high. The temperature is $360^{\circ} \mathrm{C}$, while the yield is low. The temperature $=320^{\circ} \mathrm{C}$ is selected in experiments.

To investigate the capacitance behavior of HRGO, the cyclic voltammetry (CV) and the galvanostatic chargedischarge (CD) were carried out as shown in Figures 5(a) and 5(b). It can be seen that the HRGO has higher responsive 

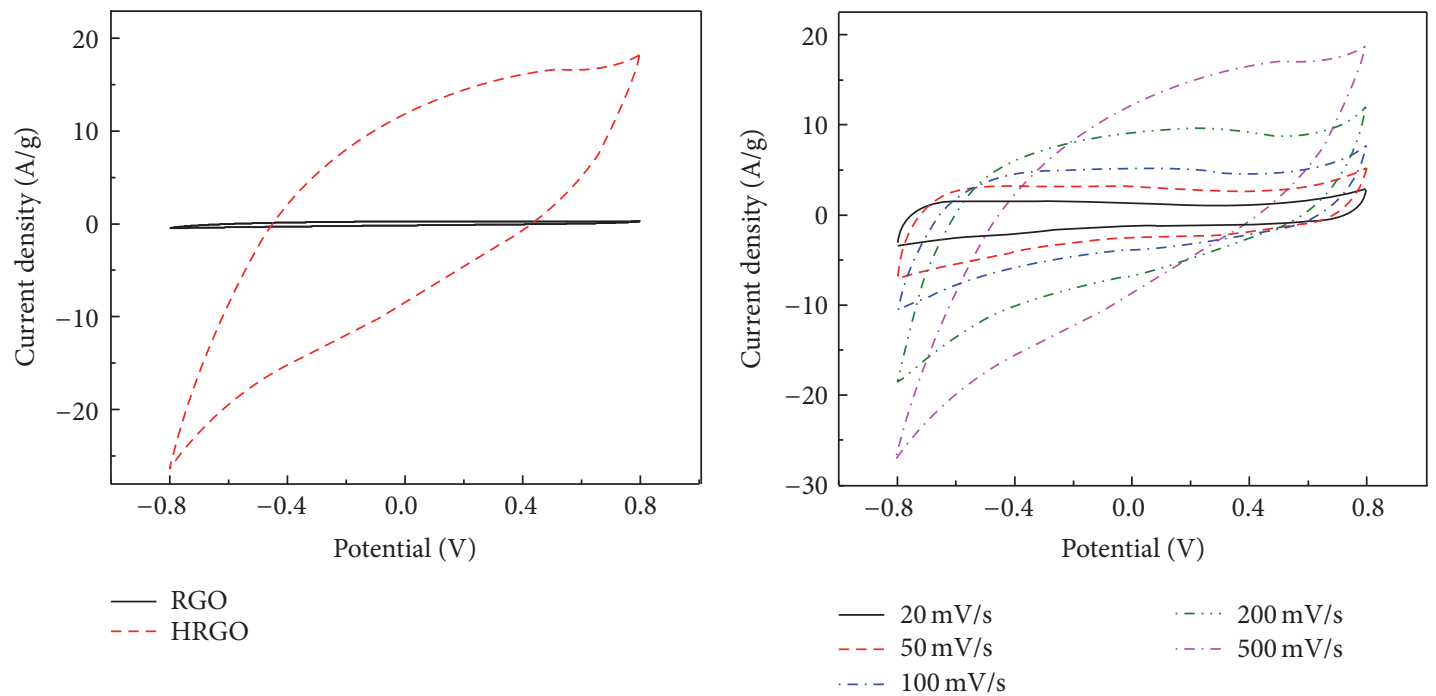

(a)

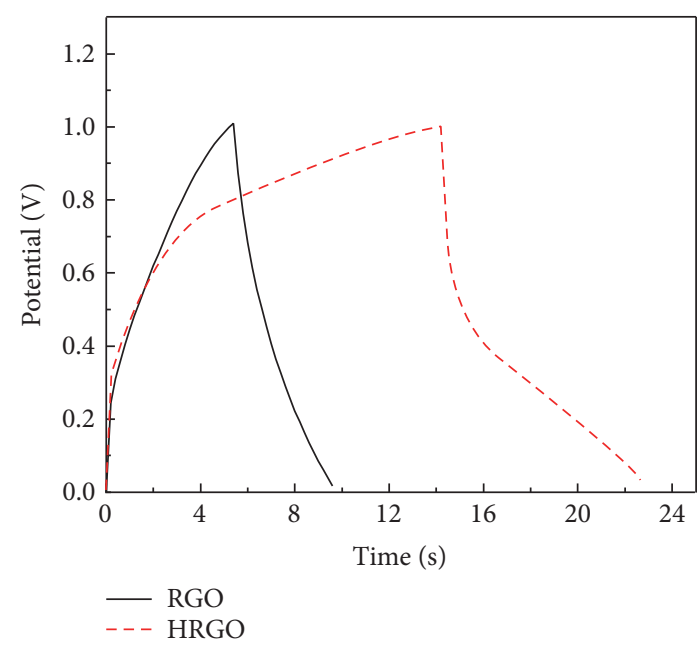

(b)

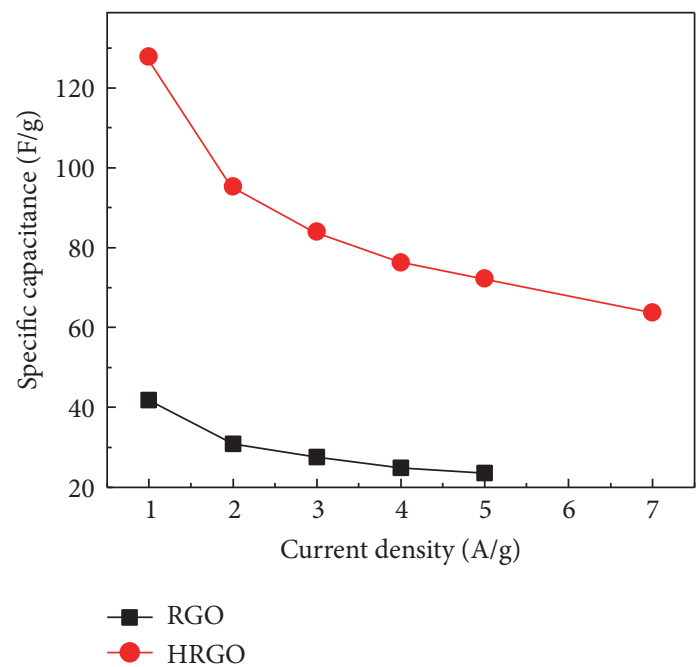

(c)

(d)

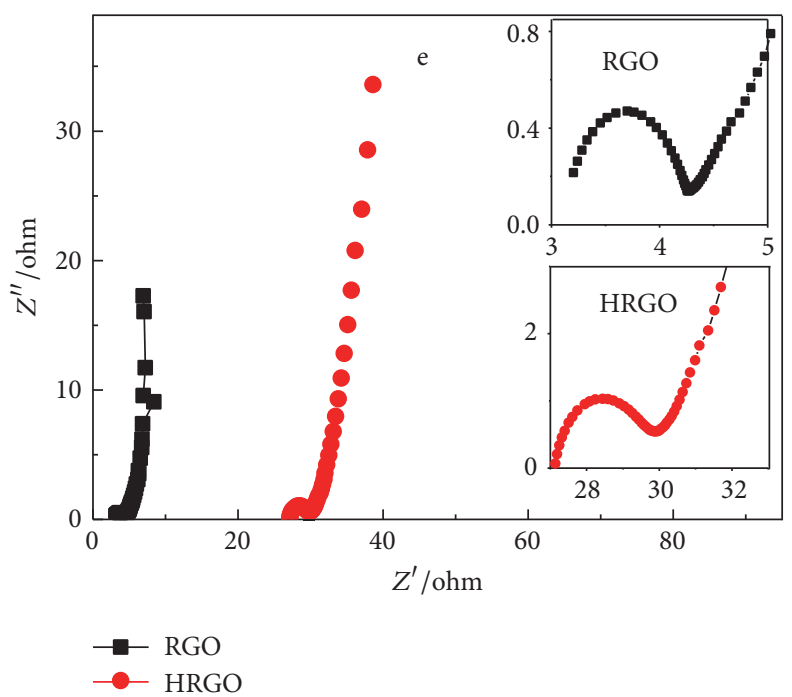

(e)

FIGURE 5: (a) CV curves of RGO and HRGO at $500 \mathrm{mV} \mathrm{s}^{-1}$; (b) CV curves of HRGO at different scanning rates; (c) the galvanostatic charge/discharge behavior of RGO and HRGO at $1 \mathrm{Ag}^{-1}$; (d) the specific capacitance of RGO and HRGO based supercapacitors at different current densities; and (e) Nyquist plots of RGO and HRGO electrodes using a sinusoidal signal of $25 \mathrm{mV}$ with the frequency range from $10 \mathrm{mHz}$ to $10 \mathrm{kHz}$. 
current density under the same potential compared to RGO at high scan rate of $500 \mathrm{mV} \mathrm{s}^{-1}$ (Figure 5(a)), which implied electron-transporting is easier and faster in the HRGO electrode. So HRGO has excellent electrochemical behavior. Further, as shown in Figure 5(b), HRGO have similar $\mathrm{CV}$ curves when the scan rate increased from $20 \mathrm{mV} \mathrm{s}^{-1}$ to $500 \mathrm{mV} \mathrm{s}^{-1}$ and the current density increased with the increase of scan rate, which indicted good rate capability of HRGO electrode. As can be seen from the CV curve, the shape is approximate rectangle, meaning electrical doublelayer capacitance mechanism for HRGO based supercapacitors. Compared to physical methods, chemical reduction methods provide graphene sheets with lots of oxygen groups, thus decrease their electrical conductivity, and result in large leakage potential [27-29]. Therefore, the effect of leakage potential was obviously observed in the two ends of the curve of HRGO in Figures 5(a) and 5(b). As described by $\mathrm{Du}$ et al. [30], polymer grafted graphene containing lots of oxygen groups have high leakage potential due to the low conductivity. Although HRGO was reduced to high level compared to general reduction methods, oxygen groups still existed in the graphene structure confirmed by the XPS curve. And, also, the CD curve shows the specific capacitance of HRGO is higher than that of RGO at the same current density $\left(1 \mathrm{~A} \mathrm{~g}^{-1}\right)$, implying the HRGO have stronger interaction with electrolyte (Figure 5(c)). From the CD curve, the leakage potential was also observed during the discharge process where the potential fell down from $1.0 \mathrm{~V}$ to $0.7 \mathrm{~V}$, also indicating the low conductivity of HRGO with oxygen containing groups. Figure 5(d) shows the specific capacitances of HRGO and RGO at different current densities. The specific capacitance of both HRGO and RGO decreased with the increase of current density, resulting from the weak electrical doublelayer. It is well-known the specific capacitance is relative to the electrical double-layer mechanism. When the current density increased, the direction of electric field switched so fast that the charge-carrying easily happened on the surface of the electrode and not deeply into the electrode, weakening electrical double-layer. It can be found that the specific capacitance of HRGO is almost three times higher than that of RGO at the same current density. The specific capacitance of RGO is $41 \mathrm{Fg}^{-1}$ while the value is $128 \mathrm{Fg}^{-1}$ for HRGO at the current density of $1 \mathrm{Ag}^{-1}$. The specific capacitance of HRGO is $72 \mathrm{Fg}^{-1}$, higher than that of RGO with $23 \mathrm{Fg}^{-1}$ when applied to the current density of $5 \mathrm{~A} \mathrm{~g}^{-1}$. And even the specific capacitance of HRGO $\left(64 \mathrm{Fg}^{-1}\right)$ at $7 \mathrm{Ag}^{-1}$ is higher than that of RGO at $1 \mathrm{Ag}^{-1}$. Therefore, HRGO retains higher specific capacitance and has a higher rate capability than RGO at high current density. The Nyquist plot of HRGO hybrid electrode shows a straight line in the low frequency region and an unconspicuous arc in the high frequency region in Figure 5(e). This high frequency loop is related to the electronic resistance inside the electrode materials. At low frequency, compared with the RGO impedance curve, the slope of the HRGO impedance curve is closer to $45^{\circ}$, indicating that the HRGO based supercapacitor is closer to the electrical double-layer capacitor characteristic. Despite high resistances of HRGO, their specific capacitance is high compared to RGO which may be attributed to high surface area and porous structure, and therefore they help to achieve the good contact between electrolyte and HRGO and improve charge mobility [31, 32].

\section{Conclusions}

In all, highly reduced graphene oxide was prepared in practical solid method. The product has few-layered sheet, and the ratio of carbon to oxygen is high. It might be more similar to graphene compared to RGO. The product has higher specific capacitance and has a higher rate capability compared to RGO at high current density. This work shows that HRGO presents unique characteristics, including facile synthesis and full reduction, which render it suitable for high-quality electronic devices, sensors, and supercapacitors, as well as fundamental studies about the chemistry and structure of graphene.

\section{Competing Interests}

The authors declare that they have no competing interests.

\section{Acknowledgments}

The authors acknowledge the financial support from Natural National Science Foundation of China (no. 51373126), the Science and Technology Department of Henan Province (no. 162300410015), the Education Department of Henan Province (no. 12B210022), the Science and Technology Bureau of Xinxiang (nos. 13SF39 and ZG15022), and Fund of the Xinxiang University (no. 15ZP05).

\section{References}

[1] P.-H. Ho, Y.-T. Liou, C.-H. Chuang et al., "Self-crack-filled graphene films by metallic nanoparticles for high-performance graphene heterojunction solar cells," Advanced Materials, vol. 27, no. 10, pp. 1724-1729, 2015.

[2] D. Geng, H. Wang, and G. Yu, "Graphene single crystals: size and morphology engineering," Advanced Materials, vol. 27, no. 18, pp. 2821-2837, 2015.

[3] A. K. Geim and K. S. Novoselov, "The rise of graphene," Nature Materials, vol. 6, no. 3, pp. 183-191, 2007.

[4] S. Park and R. S. Ruoff, "Chemical methods for the production of graphenes," Nature Nanotechnology, vol. 4, no. 4, pp. 217-224, 2009.

[5] Y. Zhu, S. Murali, M. D. Stoller et al., "Carbon-based supercapacitors produced by activation of graphene," Science, vol. 332, no. 6037, pp. 1537-1541, 2011.

[6] Z. Sun, Z. Yan, J. Yao, E. Beitler, Y. Zhu, and J. M. Tour, "Growth of graphene from solid carbon sources," Nature, vol. 468, no. 7323, pp. 549-552, 2010.

[7] P. Avouris and C. Dimitrakopoulos, "Graphene: synthesis and applications,” Materials Today, vol. 15, no. 3, pp. 86-97, 2012.

[8] T. Kuila, A. K. Mishra, P. Khanra, N. H. Kim, M. E. Uddin, and J. H. Lee, "Facile method for the preparation of water dispersible graphene using sulfonated poly(ether-ether-ketone) and its application as energy storage materials," Langmuir, vol. 28, no. 25, pp. 9825-9833, 2012. 
[9] T. Kuila, S. Bose, A. K. Mishra, P. Khanra, N. H. Kim, and J. H. Lee, "Chemical functionalization of graphene and its applications," Progress in Materials Science, vol. 57, no. 7, pp. 1061-1105, 2012.

[10] S. Stankovich, D. A. Dikin, G. H. B. Dommett et al., "Graphenebased composite materials," Nature, vol. 442 , no. 7100 , pp. $282-$ 286, 2006.

[11] B. T. Mcgrail, B. J. Rodier, and E. Pentzer, "Rapid functionalization of graphene oxide in water," Chemistry of Materials, vol. 26, no. 19, pp. 5806-5811, 2014.

[12] F. Guo, M. Creighton, Y. Chen, R. Hurt, and I. Külaots, "Porous structures in stacked, crumpled and pillared graphene-based 3D materials," Carbon, vol. 66, pp. 476-484, 2014.

[13] C. Wang, J. Zhou, J. Ni, Y. Cheng, and H. Li, "Design and synthesis of pyrophosphate acid/graphene composites with wide stacked pores for methylene blue removal," Chemical Engineering Journal, vol. 253, pp. 130-137, 2014.

[14] K. J. Stevenson, P. A. Veneman, R. I. Gearba et al., "Controlled covalent modification of epitaxial single layer graphene on $6 \mathrm{H}-\mathrm{SiC}(0001)$ with aryliodonium salts using electrochemical methods," Faraday Discussions, vol. 172, pp. 273-291, 2014.

[15] Y. Gu, H. Wu, Z. Xiong, W. Al Abdulla, and X. S. Zhao, “The electrocapacitive properties of hierarchical porous reduced graphene oxide templated by hydrophobic $\mathrm{CaCO}_{3}$ spheres," Journal of Materials Chemistry A, vol. 2, no. 2, pp. 451-459, 2014.

[16] E. J. Yoo, J. Kim, E. Hosono, H.-S. Zhou, T. Kudo, and I. Honma, "Large reversible Li storage of graphene nanosheet families for use in rechargeable lithium ion batteries," Nano Letters, vol. 8, no. 8, pp. 2277-2282, 2008.

[17] C. B. Wang, J. Ni, J. W. Zhou, J. L. Wen, and X. B. Lü, "Strategically designed porous polysilicate acid/graphene composites with wide pore size for methylene blue removal," RSC Advances, vol. 3, no. 45, pp. 23139-23145, 2013.

[18] A. C. Ferrari, J. C. Meyer, V. Scardaci et al., "Raman spectrum of graphene and graphene layers," Physical Review Letters, vol. 97, no. 18, pp. 13831-13840, 2006.

[19] Y. Y. Wang, Z. H. Ni, T. Yu et al., "Raman studies of monolayer graphene: the substrate effect," The Journal of Physical Chemistry C, vol. 112, no. 29, pp. 10637-10640, 2008.

[20] Z. H. Ni, H. M. Wang, Y. Ma, J. Kasim, Y. H. Wu, and Z. X. Shen, "Tunable stress and controlled thickness modification in graphene by annealing," ACS Nano, vol. 2, no. 5, pp. 1033-1039, 2008.

[21] D. C. Elias, R. R. Nair, T. M. G. Mohiuddin et al., "Control of graphene's properties by reversible hydrogenation: evidence for graphane," Science, vol. 323, no. 5914, pp. 610-613, 2009.

[22] C. B. Wang, J. W. Zhou, and L. L. Chu, "Chlorine-functionalized reduced graphene oxide for methylene blue removal," RSC Advances, vol. 5, no. 65, pp. 52466-52472, 2015.

[23] H. Roghani-Mamaqani, V. Haddadi-Asl, K. Khezri, and M. Salami-Kalajahi, "Edge-functionalized graphene nanoplatelets with polystyrene by atom transfer radical polymerization: grafting through carboxyl groups," Polymer International, vol. 63, no. 11, pp. 1912-1923, 2014.

[24] W. B. Wan, L. L. Li, Z. B. Zhao et al., "Graphene oxide: ultrafast fabrication of covalently cross-linked multifunctional," Advanced Functional Materials, vol. 24, no. 31, pp. 4915-4921, 2014.

[25] K. Spyrou, G. Potsi, E. K. Diamanti et al., "Towards novel multifunctional pillared nanostructures: effective intercalation of adamantylamine in graphene oxide and smectite clays,"
Advanced Functional Materials, vol. 24, no. 37, pp. 5841-5850, 2014.

[26] Y. X. Xu, K. X. Sheng, C. Li, and G. Q. Shi, "Self-assembled graphene hydrogel via a one-step hydrothermal process," ACS Nano, vol. 4, no. 7, pp. 4324-4330, 2010.

[27] W. T. Deng, X. B. Ji, M. Gómez-Mingot, F. Lu, Q. Y. Chen, and C. E. Banks, "Graphene electrochemical supercapacitors: the influence of oxygen functional groups," Chemical Communications, vol. 48, no. 22, pp. 2770-2772, 2012.

[28] H. Tateishi, M. Koinuma, S. Miyamoto et al., "Effect of the electrochemical oxidation/reduction cycle on the electrochemical capacitance of graphite oxide," Carbon, vol. 76, no. 9, pp. 40-45, 2014.

[29] Y. J. Oh, J. J. Yoo, Y. I. Kim et al., “Oxygen functional groups and electrochemical capacitive behavior of incompletely reduced graphene oxides as a thin-film electrode of supercapacitor," Electrochimica Acta, vol. 116, no. 2, pp. 118-128, 2014.

[30] F.-P. Du, J.-J. Wang, C.-Y. Tang et al., "Water-soluble graphene grafted by poly(sodium 4-styrenesulfonate) for enhancement of electric capacitance," Nanotechnology, vol. 23, no. 47, Article ID 475704, 2012.

[31] Z. Bo, W. G. Zhu, X. Tu et al., "Instantaneous reduction of graphene oxide paper for supercapacitor electrodes with unimpeded liquid permeation," The Journal of Physical Chemistry C, vol. 118, no. 25, pp. 13493-13502, 2014.

[32] H. W. Chang, Y. R. Lu, J. L. Chen et al., "Electrochemically activated reduced graphene oxide used as solid-state symmetric supercapacitor: an X-ray absorption spectroscopic investigation," The Journal of Physical Chemistry C, vol. 120, no. 39, pp. 22134-22141, 2016. 

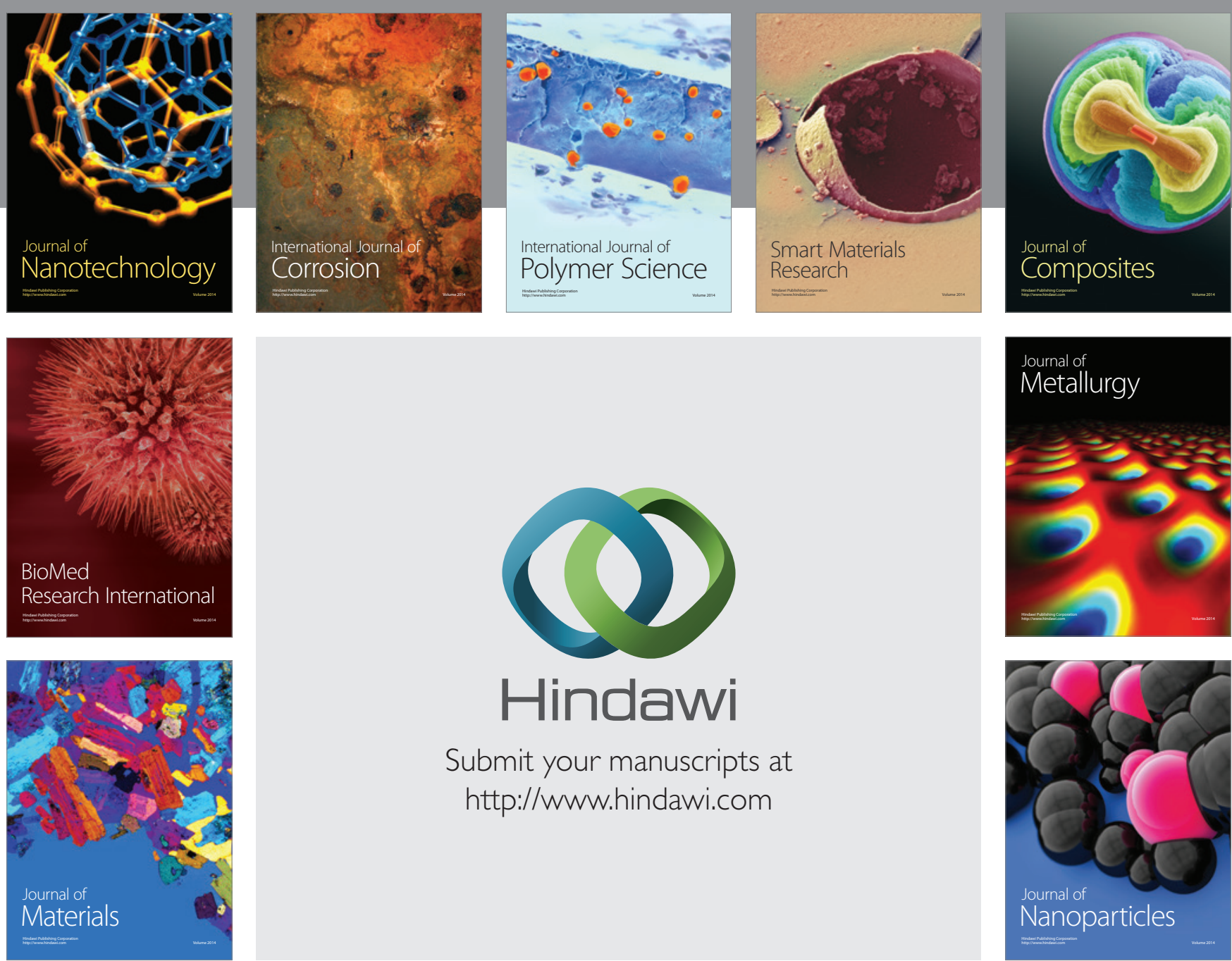

\section{Hindawi}

Submit your manuscripts at

http://www.hindawi.com

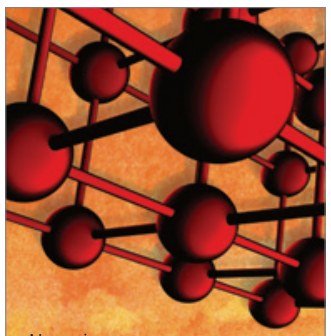

Materials Science and Engineering
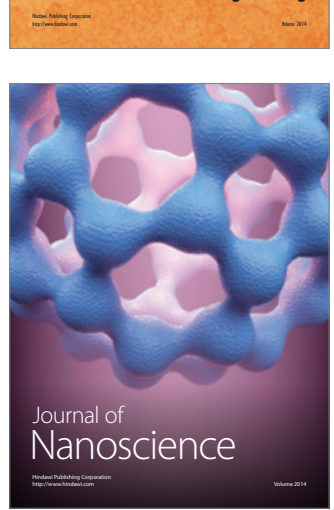
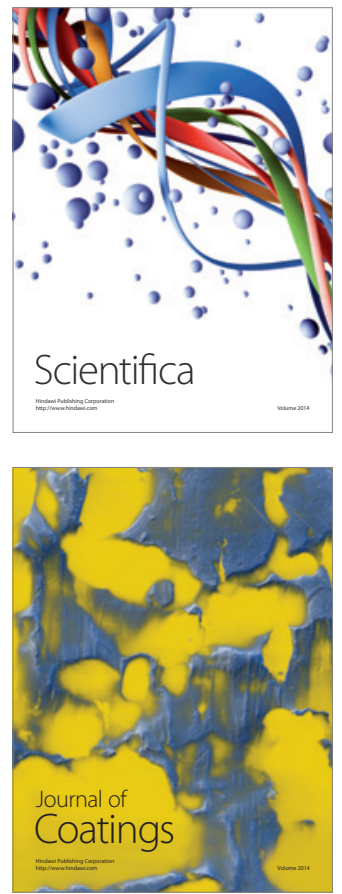
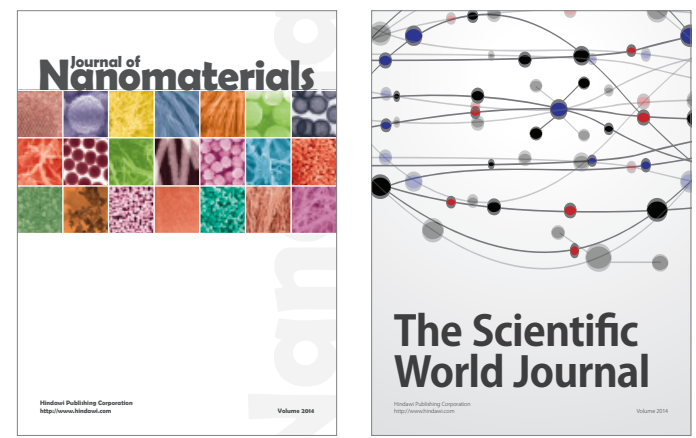

The Scientific World Journal
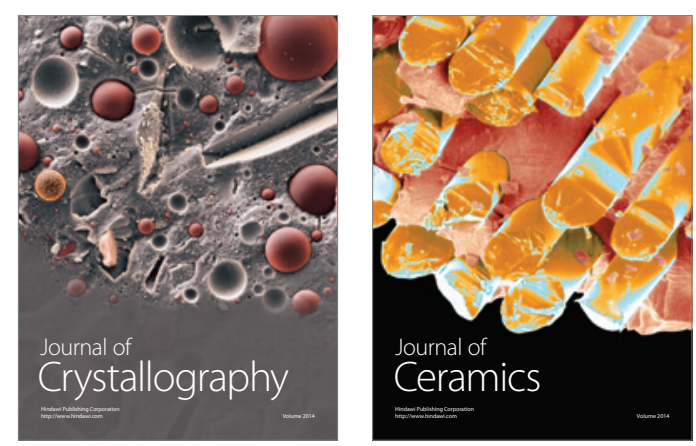
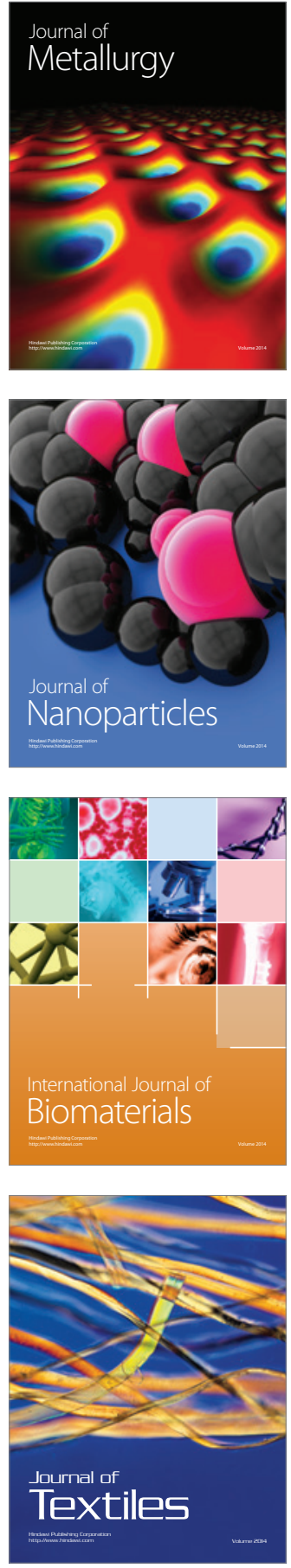